\title{
Optimal margin distance of bullectomy for primary spontaneous pneumothorax reduces postoperative recurrence
}

\author{
Kenji Tsuboshima*, Yasumi Matoba, Teppei Wakahara \\ Department of Thoracic Surgery and Pneumothorax Center, Takasago Municipal Hospital, Takasago, Japan \\ Contributions: (I) Conception and design: K Tsuboshima; (II) Administrative support: Y Matoba; (III) Provision of study materials or patients: K \\ Tsuboshima; (IV) Collection and assembly of data: All authors; (V) Data analysis and interpretation: K Tsuboshima; (VI) Manuscript writing: All \\ authors; (VII) Final approval of manuscript: All authors. \\ Correspondence to: Kenji Tsuboshima, MD. Pneumothorax Research Center and Division of Thoracic Surgery, Nissan Tamagawa Hospital, 4-8-1 Seta \\ Setagaya-ku, Tokyo 158-0095, Japan. Email: drniwatori@smn.enjoy.ne.jp.
}

\begin{abstract}
Background: For primary spontaneous pneumothorax, bullectomy using autosutures is the standard procedure. Despite performing various methods for preventing postoperative recurrence, it remains relatively high. Although considering the margin distance of bullectomy is important, no argument has been discussed for the optical margin distance until now. Hence, we evaluated the optimal margin distance for bullectomy of spontaneous pneumothorax, bullectomy to reduce postoperative recurrence.

Methods: Between March 2015 and May 2018, 91 eligible candidates from 142 cases, who underwent video-assisted thoracoscopic bullectomy for spontaneous pneumothorax, bullectomy, were evaluated. We analysed the factors preventing postoperative recurrence, including the margin distance using the inverseprobability of treatment weighted method by propensity score was used to adjust for heterogeneous patient backgrounds.

Results: The patients' median age was 20 years (range, 14-86 years). Postoperative recurrences occurred in 8 cases $(8.8 \%)$. The margin distance was $9.1 \pm 5.0 \mathrm{~mm}$. The cut-off point of the margin distance was defined as $5.0 \mathrm{~mm}$. The inverse-probability of treatment weighted method indicated that a margin distance $\geq 5.0 \mathrm{~mm}$ significantly prevented postoperative recurrence $(\mathrm{P}=0.0076$, hazard ratio $=0.18)$.

Conclusions: This study suggests that a margin distance of $\geq 5.0 \mathrm{~mm}$ for bullectomy of PSP can reduce postoperative recurrence.
\end{abstract}

Keywords: Primary spontaneous pneumothorax (PSP); recurrence; margin distance; propensity score

Submitted Jul 31, 2019. Accepted for publication Nov 12, 2019.

doi: $10.21037 /$ jtd.2019.12.06

View this article at: http://dx.doi.org/10.21037/jtd.2019.12.06

\section{Introduction}

For primary spontaneous pneumothorax (PSP), videoassisted thoracoscopic surgery (VATS) bullectomy using autosutures is the standard surgical procedure (1-4). Although various procedures, such as visceral pleural reinforcement by absorbable covering sheet, chemical, or mechanical pleurodesis, to prevent postoperative recurrence of PSP have been performed, it remains relatively high
(1-6). Therefore, a different viewpoint to resolve this problem is necessary. Wedge resection using autosutures is a procedure used for non-small cell lung cancer (NSCLC) $(7,8)$. Some reports have commented on the sufficient margin distance to reduce postoperative local recurrence (7-9). Since intrapulmonary bulla is not identified through the thoracoscopic view precisely, an insufficient margin distance of bullectomy may be the cause of residual bulla, which is related to postoperative recurrence. There are

\footnotetext{
* Pneumothorax Research Center and Division of Thoracic Surgery, Nissan Tamagawa Hospital, Tokyo, Japan at present.
} 


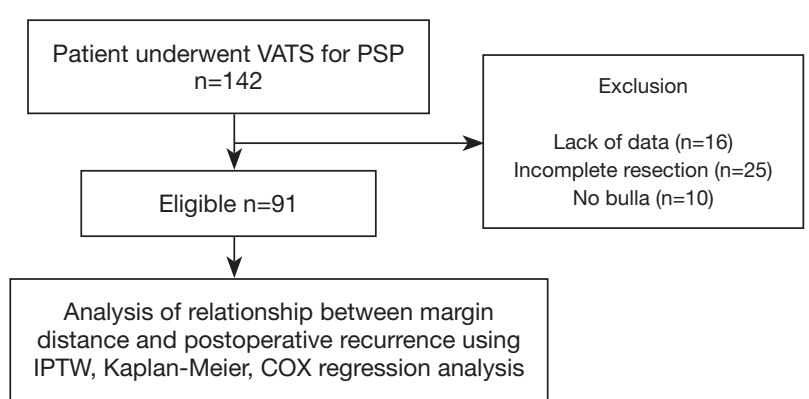

Figure 1 Details of enrolment in the study. BMI, body mass index; HRCT, high resolution computed tomography; IPTW, InverseProbability of Treatment Weighted; PSP, primary spontaneous pneumothorax; VATS, video-assisted thoracoscopic surgery.

some guidelines for the management of PSP (10-12). However, no argument has been discussed for the optical margin distance for bullectomy to prevent postoperative recurrence, unlike NSCLC. Therefore, we evaluated the optimal margin distance for bullectomy of PSP to reduce postoperative recurrence.

\section{Methods}

Between March 2015 and May 2018, we enrolled 142 consecutive PSP patients who underwent VATS bullectomy using autosutures in our hospital. PSP was defined as a pneumothorax without underlying lung diseases. Cases with a lack of clinical data, cases without complete resection of bullae, or cases without confirmed bullae were excluded. For cases without complete resection due to hilar, interlobar or multiple bullae, additional abrasion or covering was performed. In total, 91 cases were retrospectively evaluated (Figure 1). The factors preventing postoperative recurrence were evaluated using the Kaplan-Meier method and the inverse-probability of treatment weighted (IPTW) method by propensity score was also used to adjust for heterogeneous patient backgrounds. The 11 predicted factors preventing postoperative recurrence consisted of the following: age, gender, operation side, smoking habit, history of ipsilateral surgery, body mass index (BMI), bulla size, bulla type, pleural reinforcement by covering absorbable sheet, use of fibrin glue, and the margin distance of bullectomy. For margin distance, we defined the cut-off point of $5.0 \mathrm{~mm}$, which is approximately half of the short diameter of the autosuture. The cut-off points of other continuous variables such as age and BMI were defined according to the median. Bulla size was defined as the

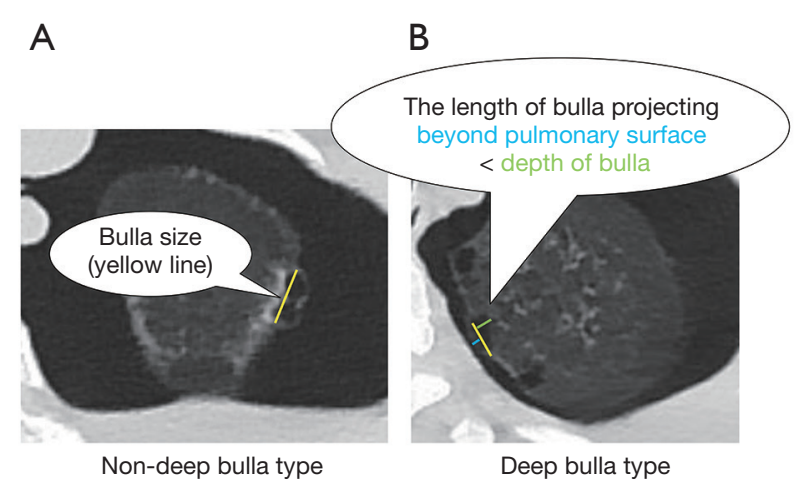

Figure 2 (A) Definition of bulla size, and (B) definition of deep bulla on high-resolution computed tomography (HRCT).

maximum root length of bulla by high resolution computed tomography (HRCT) in Figure 2. For bulla size, we defined the cutoff point of $10.0 \mathrm{~mm}$, which is approximately the median size. For bulla type, deep bulla was defined as bulla in which the internal depth was larger than the external size in Figure 2. Bulla, other than deep bulla, was defined as non-deep bulla. As an exploratory study, four subgroupsbulla size $<10, \geq 10 \mathrm{~mm}$, non-deep bulla, and deep bulla were evaluated using univariate analysis.

The patients underwent VATS according to the following indications: ipsilateral PSP recurrence, a persistent air leak after chest tube drainage, contralateral PSP, hemopneumothorax, tension pneumothorax, and patient request. All procedures were performed by a single surgical team. The patient was placed in a lateral position under general anaesthesia with ventilation of one lung. According to the difficulty of VATS, the choice of procedure (singleor multi-port) was made as we previously described $(13,14)$. The bullae to be resected were decided from preoperative HRCT and intraoperative findings. The bullae were resected by autosuture (Echelon FLEX, JJKK, USA or Endo-GIA, Covidien, USA, depending on the surgeon's decision) with a margin distance, which was decided by the surgeon when the team was certain that no residual bullae were present through the thoracoscopic view.

The margin distance was evaluated by a ruler in the operating room using the resected lung and was defined as the distance between the staple line and the nearest bulla (Figure 3). If the bullae were far apart, the resected lung had multiple parts. In such cases, the shortest margin distance was recorded.

Subsequently, we performed a water submersion test, which involved inflating the lung under $20 \mathrm{~cm}$ of water 


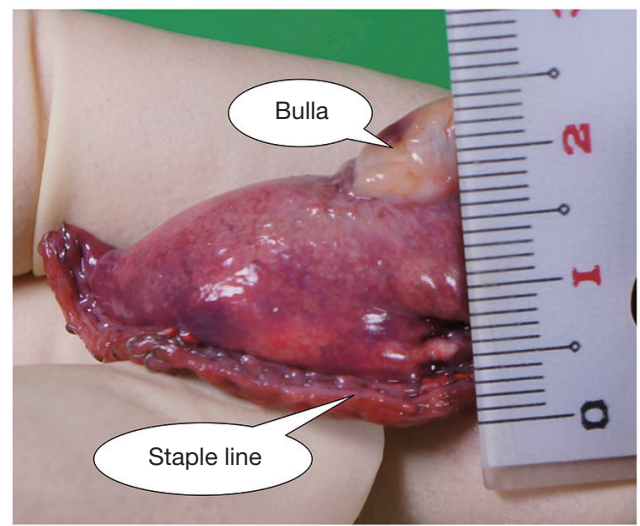

Figure 3 Method of measurement for margin distance of bullectomy.

pressure, to detect any persistent air leakage. When an air leak was detected, additional resection or fibrin glue was used. The staple line was covered with oxidised regenerated cellulose (ORC) and/or polyglycolic acid (PGA) sheet to prevent postoperative recurrence of PSP without suspected intrathoracic infection. If necessary, fibrin glue was also used to fix the absorbable covering sheets on the visceral pleura. Depending on the surgeon's preference, fragility of the lung, presence of air leakage during surgery, covering materials and fibrin glue were selected. If there were no clear findings of intrathoracic infection, a pleural coating using $50 \%$ glucose was performed, as we previously described, to reduce postoperative recurrence (1). After placing a chest tube in the intrathoracic cavity, the wound was closed. Then, the chest tube was connected to an aspiration system and negative suction of $10 \mathrm{cmH}_{2} \mathrm{O}$ was applied.

We removed the chest tube after postoperative day one, when the air leak was stopped, and no bloody effusion was detected regardless of the volume of effusion. Even for asymptomatic patients, chest radiography or computed tomography was performed every 3 months postoperatively for 12 months. If symptoms appeared, patients visited our institution without reservation. Regardless of the intervention, even a case of minimal lung collapse confirmed on imaging was considered as recurrence. The follow-up was continued for 1 year after surgery; those who could not be followed up till then were censored on the date of the last follow-up. The follow-up period was started from the date of operation. Morbidity or mortality associated with our procedures was evaluated according to The Japan Clinical Oncology Group postoperative complication criteria based on Clavien-Dindo classification (15). According to the criteria, grade IIIb or more is needed for invasive procedures under general anaesthesia; therefore, the adverse events that corresponded to grade IIIb or more were defined as severe morbidity.

Statistical analyses were performed using the Welch test and the Fisher's exact test. Continuous variables were represented as mean \pm standard deviation. Univariate analysis of the postoperative recurrence rate for PSP was estimated based on the Kaplan-Meier method and compared by the log-rank test. For a propensity score analysis, the Cox regression analysis using IPTW method was performed. A P value of $<0.05$ indicated statistical significance. The hazard ratio (HR) and $95 \%$ confidence intervals (CIs) were calculated for each variable using Cox regression analysis. EZR software package (http://www. jichi.ac.jp/saitama-sct/SaitamaHP.files/statmed.html) was used for all statistical analyses. This study was approved by the institutional review board of Takasago Municipal Hospital (No. 2018-3), and informed consent was obtained through the hospital website with an opt-out option.

\section{Results}

The patients' median age was 20 years (range, $14-86$ years; Table 1). Smoking habit was present in $26(28.6 \%)$ cases (Table 1). Of the total, $10(11.0 \%)$ cases had a history of ipsilateral surgery (Table 1). Deep bullae were detected in 17 cases (18.7\%; Table 1), while the median bulla size was $11.2 \mathrm{~mm}$ (Table 1). The absorbable covering sheet and the fibrin glue were used for $83(91.2 \%)$ and 7 (7.7\%) cases, respectively (Table 1$)$. There were $8(8.8 \%)$ cases of postoperative recurrence (Table 1). Two cases needed reoperation, and 6 cases were observed conservatively (Table 2). No cases of air leak point that caused recurrence were found (Table 2). The margin distance of bullectomy was $9.1 \pm 5.0 \mathrm{~mm}$ (Table 1). The mean follow-up period was $271.3 \pm 219.1$ days (Table 1 ).

From the univariate analysis using the log-rank test, a margin distance $\geq 5.0 \mathrm{~mm}$ was determined to be significant in preventing postoperative recurrence of PSP (Table 3). Between the two groups, for a margin distance cutoff point of $5.0 \mathrm{~mm}$, no significant differences in the backgrounds were found (Table 4). Kaplan-Meier method and the Cox regression analysis showed the margin distance $\geq 5.0 \mathrm{~mm}$ as a significant factor preventing postoperative recurrence of PSP ( $\mathrm{P}=0.012$; HR $=0.20 ; 95 \%$ CI, 0.050-0.81; Table 3, Figure 4). Based on the IPTW, the margin distance 
Table 1 Clinical characteristics of patients with PSP who underwent VATS

\begin{tabular}{|c|c|c|c|}
\hline Variables & Total $(n=91)$ & $\begin{array}{l}\text { Postoperative recurrence } \\
\text { case }(n=8)\end{array}$ & $\begin{array}{c}\text { Non-postoperative recurrence } \\
\text { case }(n=83)\end{array}$ \\
\hline Age (years), median [range] & 20 [14-86] & 17 [15-34] & 20 [14-86] \\
\hline Male, n (\%) & $83(91.2)$ & $8(100.0)$ & $75(90.4)$ \\
\hline Smoking habit, n (\%) & $26(28.6)$ & $1(12.5)$ & $25(30.1)$ \\
\hline BMI, median [range] & $19.4(14.0-25.1)$ & $18.4(15.7-19.6)$ & $19.6(14.1-25.1)$ \\
\hline Bulla size $(\mathrm{mm})$, median [range] & $11.2(2.1-49.2)$ & $13.3(4.7-18.3)$ & $11.0(2.1-49.2)$ \\
\hline Deep bulla, n (\%) & $17(18.7)$ & $1(12.5)$ & $16(19.3)$ \\
\hline Margin distance (mm) & $9.1 \pm 5.0$ & $7.6 \pm 5.5$ & $9.2 \pm 5.0$ \\
\hline Follow-up period (days) & $271.3 \pm 219.1$ & $321.5 \pm 169.8$ & $268.4 \pm 223.4$ \\
\hline
\end{tabular}

BMI, body mass index; PSP, primary spontaneous pneumothorax; VATS, video-assisted thoracoscopic surgery.

Table 2 The detail of postoperative recurrent cases

\begin{tabular}{|c|c|c|c|c|c|c|c|c|c|}
\hline No & Age & Gender & Side & $\begin{array}{l}\text { Deep } \\
\text { bulla }\end{array}$ & $\begin{array}{c}\text { Margin } \\
\text { distance }(\mathrm{mm})\end{array}$ & $\begin{array}{l}\text { Follow-up } \\
\text { period (days) }\end{array}$ & New bulla on $\mathrm{CT}$ & Intervention & Identified lesion \\
\hline 1 & 20 & Male & Left & No & 9 & 184 & Unknown & Observation & Unknown \\
\hline 2 & 17 & Male & Right & Yes & 2 & 196 & Unknown & Observation & Unknown \\
\hline 3 & 21 & Male & Left & No & 12 & 342 & Unknown & Observation & Unknown \\
\hline 6 & 17 & Male & Right & No & 4 & 595 & Unknown & Observation & Unknown \\
\hline 7 & 16 & Male & Right & No & 15 & 203 & Near the resected lung & Reoperation & $\begin{array}{l}\text { New bulla near the } \\
\text { resected lung }\end{array}$ \\
\hline 8 & 15 & Male & Right & No & 2 & 148 & Apart from resected lung & Reoperation & $\begin{array}{l}\text { New bulla apart from } \\
\text { resected lung }\end{array}$ \\
\hline
\end{tabular}

CT, computed tomography.

$\geq 5.0 \mathrm{~mm}$ was a significant factor preventing postoperative recurrence of PSP $(\mathrm{P}=0.0076$; $\mathrm{HR}=0.18$; 95\% CI, 0.052 $0.64 ;$ Table 5). In the subgroup analysis, the margin distance $\geq 5.0 \mathrm{~mm}$ was a significant factor preventing postoperative recurrence of PSP with deep bulla and bulla size $\geq 10 \mathrm{~mm}$ ( $\mathrm{P}=0.0082$ and $\mathrm{P}=0.011$, relatively, Table 6). However, it was not a significant factor for non-deep bulla and bulla size
$<10 \mathrm{~mm}$ (no data). No severe morbidity and no mortality were found.

\section{Discussion}

For PSP, VATS bullectomy using autosutures is one of the standard surgical procedures. However, as VATS bullectomy 
Table 3 Univariate analysis of factors preventing postoperative recurrence of PSP using Kaplan-Meier method

\begin{tabular}{|c|c|c|c|}
\hline Variables & Postoperative recurrence rate (\%) & $\mathrm{HR}(95 \% \mathrm{Cl})$ & $P$ value \\
\hline Gender (male vs. female) & 9.6 vs. 0.0 & - & 0.42 \\
\hline Smoking habit (yes vs. no) & 3.8 vs. 10.8 & $0.49(0.059-4.07)$ & 0.50 \\
\hline Operation side (right vs. left) & 15.4 vs. 3.8 & $0.24(0.047-1.20)$ & 0.054 \\
\hline BMI (<19 vs. $\geq 19)$ & 12.5 vs. 5.9 & $0.55(0.13-2.33)$ & 0.38 \\
\hline Deep bulla (yes vs. no) & 5.9 vs. 9.5 & $0.61(0.072-5.07)$ & 0.64 \\
\hline Bulla size (<10 vs. $\geq 10 \mathrm{~mm}$ ) & 5.0 vs. 11.8 & $0.47(0.091-2.38)$ & 0.35 \\
\hline The reinforcement of pleura (yes vs. no) & 9.6 vs. 0.0 & - & 0.19 \\
\hline
\end{tabular}

$\mathrm{BMI}$, body mass index; $\mathrm{Cl}$, confidence interval; HR, hazard ratio; PSP, primary spontaneous pneumothorax.

Table 4 Clinical characteristics of the patients with PSP who underwent VATS according margin distance

\begin{tabular}{|c|c|c|c|}
\hline Variables & Margin distance $<5$ mm $(n=16)$ & Margin distance $\geq 5 \mathrm{~mm}(\mathrm{n}=75)$ & $P$ value \\
\hline Male, n (\%) & $15(93.8)$ & $68(90.7)$ & 1.00 \\
\hline Smoking habit, n (\%) & $5(31.2)$ & $21(28.0)$ & 0.77 \\
\hline Right side, n (\%) & $7(43.8)$ & $32(42.7)$ & 1.00 \\
\hline BMI, median [range] & $20.4[15.5-25.1]$ & $19.1[14.1-24.6]$ & 0.15 \\
\hline Bulla size (mm), median [range] & $17.2[6.0-37.0]$ & $10.0[2.1-49.2)$ & 0.072 \\
\hline Deep bulla, n (\%) & $2(12.5)$ & $15(20.0)$ & 0.73 \\
\hline The reinforcement of pleura, $\mathrm{n}(\%)$ & $16(100.0)$ & $67(89.3)$ & 0.34 \\
\hline
\end{tabular}

BMI, body mass index; PSP, primary spontaneous pneumothorax; VATS, video-assisted thoracoscopic surgery.

alone has a high recurrence rate of $16.3-24.1 \%(2,3)$, the following four methods have been primarily considered to reduce the postoperative recurrence rate. First, absorbable covering sheets for reinforcement of the visceral pleura around the staple line is thought to be one of the effective techniques in preventing postoperative recurrence $(2,4)$. In a prospective randomised controlled study by Lee $e t$ al., it was shown that the use of fibrin glue and absorbable ORC mesh for reinforcement of the staple line after VATS bullectomy for PSP was comparable to mechanical pleurodesis in terms of preventing postoperative recurrence $(13.8 \%$ vs. $14.2 \%$ in 1,414 cases at 11 institutions) (4). Hirai et al. and Hong et al. reported that VATS bullectomy with PGA sheet and fibrin glue reduced the postoperative recurrence rate to $3.3 \%$ in 279 cases and $8.6 \%$ in 116 cases, relatively. This method reduced postoperative recurrence more than bullectomy alone $(2,3)$. Second, mechanical pleurodesis, such as parietal pleural abrasion or pleurectomy, has been performed. Rena $e t$ al. reported the feasibility and safety of intraoperative mechanical pleural abrasion for PSP in 220 cases in a randomised controlled trial, where the postoperative recurrence rate was $4.2 \%$ (5). Third, chemical 
pleurodesis using agents, such as talc, glucose, OK432, and minocycline, has been performed. Cardillo et al. described the feasibility of VATS bullectomy and talc poudrage for postoperative recurrence of PSP retrospectively in 861 cases. The recurrence rate was $1.73 \%$ (6). Forth, hybrid procedures, which combined the above three methods, have

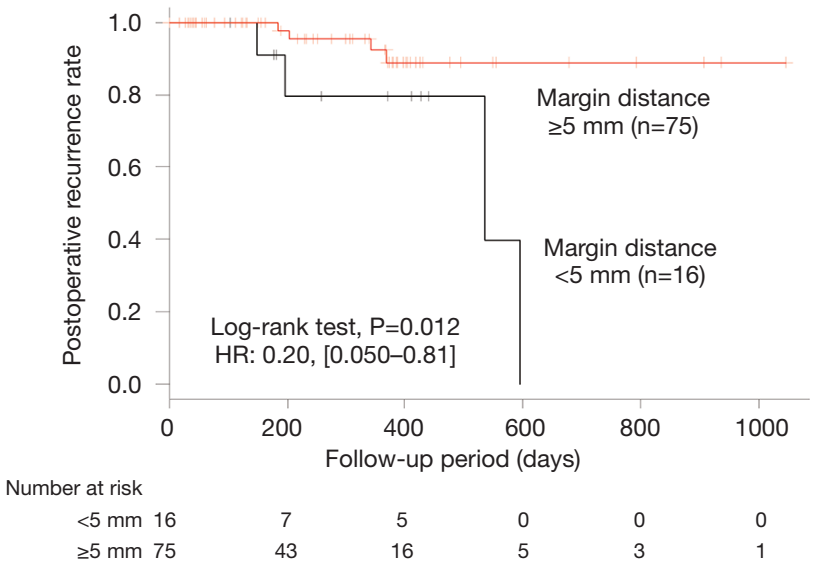

Figure 4 Kaplan-Meier survival curves of postoperative recurrence rate for primary spontaneous pneumothorax considering the margin distance. been performed. We described a significant reduction in the rate of postoperative spontaneous pneumothorax recurrence by the pleural coating method using $50 \%$ glucose and absorbable sheet in 376 cases. The recurrence rate was $8.3 \%$. The Cox regression analysis with propensity score matching and the IPTW method indicated that the procedure significantly prevented postoperative recurrence $(\mathrm{P}=0.014$, $\mathrm{HR}=0.15$ and $\mathrm{P}=0.0038, \mathrm{HR}=0.23$, respectively) (1). Nevertheless, postoperative recurrence was relatively common regardless of these reinforcements (1.22-14.2\%) (1-6). Therefore, a different viewpoint to resolve this problem was necessary.

Although it is essential to consider the margin distance of bullectomy for PSP, it was decided according to the experience of the surgeon. Meanwhile, for cases with NSCLC, which is a contraindication for lobectomy due to low respiratory function, wedge resection using autosutures has been performed as a compensatory limited resection. Some studies have reported the optimal margin distance of wedge resection of NSCLC. Sawabata $e t$ al. reported that malignant negative margins were found when the margin distance was greater than the maximum tumour diameter for peripheral NSCLC (7). Mohiuddin et al. described that increasing the margin distance $\leq 15 \mathrm{~mm}$ significantly

Table 5 IPTW method using propensity score to assess the factor preventing postoperative recurrence of PSP

\begin{tabular}{llll}
\hline Variables & HR & $\mathrm{P}$ value & $95 \% \mathrm{Cl}$ \\
\hline Margin distance $\geq 5 \mathrm{~mm}$ & 0.18 & 0.0076 & $0.052-0.64$ \\
\hline
\end{tabular}

$\mathrm{Cl}$, confidence interval; HR, hazard ratio; IPTW, inverse-probability of treatment weighted; PSP, primary spontaneous pneumothorax.

Table 6 Subgroup analysis preventing postoperative recurrence of PSP using Kaplan-Meier method

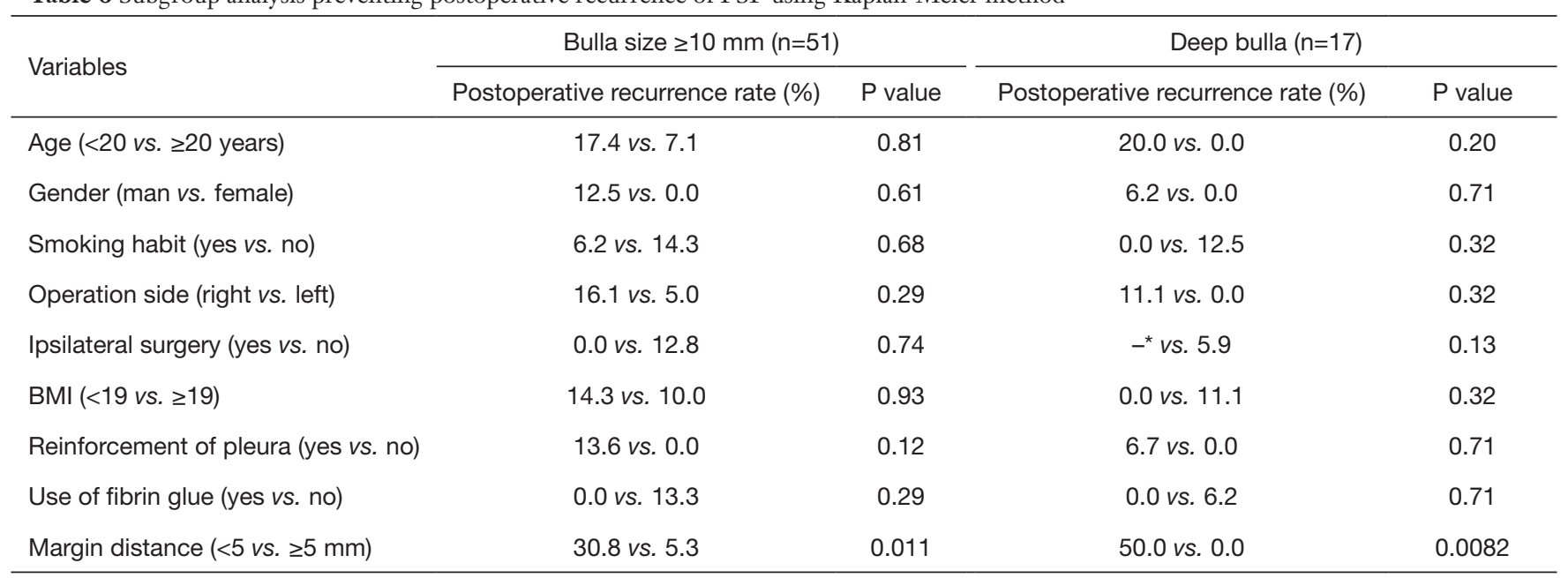

${ }^{*}$, no ipsilateral surgery. BMI, body mass index; PSP, primary spontaneous pneumothorax. 
reduced the local recurrence risk for wedge resection for small $(\leq 2 \mathrm{~cm})$ NSCLC (8). Wolf et al. showed that a margin distance $>9 \mathrm{~mm}$ was associated with the longest recurrencefree survival in patients undergoing wedge resection for NSCLC tumours $\leq 2 \mathrm{~cm}(9)$.

Perhaps, Surgeons may differ in their preference for minimal or larger resections. Minimal resections may favour better postoperative respiratory function, while large resections are suitable for complete resection of intrapulmonary residual bulla and missed bulla to prevent postoperative recurrence. Therefore, no optimal margin distance may have been reported, unlike NSCLC. There are fewer problems of symptomatic respiratory functional disorder for PSP postoperatively and postoperative recurrence is a severer problem. Although there are some guidelines for the management of PSP, the optimal margin distance of bullectomy for PSP has not been described (10-12). As intrapulmonary bulla is not precisely identified through the thoracoscopic view, insufficient margin distance of bullectomy may be the cause of residual bulla, which is related to postoperative recurrence. Thus, we evaluated the effect of the margin distance of bullectomy on postoperative recurrence.

The cut-off point of the margin distance was defined as $5.0 \mathrm{~mm}$. Our study revealed the margin distance $\geq 5.0 \mathrm{~mm}$ as the significant factor preventing postoperative recurrence for PSP. It was thought that age and smoking habit affected postoperative recurrence $(1,2,4)$. The margin distance is a factor that could be controlled by thoracic surgeons intraoperatively, unlike age and past smoking habit that we could not control. The determined margin distance of $5.0 \mathrm{~mm}$ was about half of the short diameter of the autosuture. If no bulla is compressed by autosuture, it was thought that margin distance $\geq 5.0 \mathrm{~mm}$ could be confirmed without other devices, such as a ruler, in the thoracic cavity. However, unlike the intrathoracic view, the margin distance tended to be underestimated empirically. Compared to the evaluation with the ruler for resected lung, the compression of autosutures may expand the margin distance from the resected lung. Therefore, a little additional margin distance may be needed than the predicted margin distance from thoracoscopic view. In the present study, there were 16 cases with the margin distance $<5.0 \mathrm{~mm}$. This was a relatively large number. This may be primarily due to the intraoperative overestimation of the margin distance. In other cases, since there were numerous bullae and it was necessary to resect a large volume of the lung. We intentionally made a minimal margin distance in case an unexpected small bulla near the staple line existed in the resected lung.

The most suitable mechanism contributing to the reduction of the postoperative recurrence rate has not yet been clarified. Although we can identify the range of extrapulmonary bulla through the thoracoscopic view, we cannot identify the range of intrapulmonary bulla and we may miss the small bulla. Therefore, we believe that the optimal margin distance reduced the intrapulmonary residual bulla and undetected bulla, which may cause the development of the postoperative bulla neogenesis and recurrence. In this hypothesis, it may be useful that the diagnosis from the intraoperative frozen section of the resected lung margin is performed to predict the complete resection of the intrapulmonary bulla. The bulla size and type may affect intrapulmonary bulla. Thus, we evaluated subgroup analysis as an exploratory study. For subgroups of bulla size $\geq 10 \mathrm{~mm}$ and deep bulla, the relationship between margin distance $\geq 5.0 \mathrm{~mm}$ and a significantly lower postoperative recurrence was shown. This result might suggest that a sufficient margin distance prevented intrapulmonary residual bulla near the staple line and postoperative recurrence was also reduced.

We think that postoperative pneumothorax recurrence is not only caused by margin distance but also by new bulla apart from the resected site (16). In fact, for case 8 in Table 2, new bulla apart from the resected site may be the cause of the recurrence. In such a case, although margin distance may be irrelevant to postoperative recurrence, on the basis of our results, we think that margin distance is valuable for predicting postoperative pneumothorax recurrence.

Due to the retrospective nature of our study, the patient backgrounds were heterogeneous. Recently, for a retrospective study, the IPTW method by propensity score was used to adjust patient backgrounds $(1,14,17)$. Hence, we also used the IPTW method to evaluate the relationship between the margin distance and the postoperative recurrence, in addition to the Kaplan-Meier method and Cox regression analysis.

Our study has some limitations. It was carried out at a single institution, and it had a retrospective design. Despite performing the IPTW method to adjust the heterogeneous patient backgrounds, unknown factors that might affect the postoperative recurrence could not be adjusted. Therefore, we think a prospective study including patients from multiple institutions is required. Although we evaluated the subgroup analysis as an exploratory study considering the 
bulla size and type, the number of cases was very small. In a future study, we plan to recruit a larger number of cases to circumvent these issues.

\section{Conclusions}

This study suggested that an optical margin distance of $\geq 5.0 \mathrm{~mm}$ in bullectomy reduced the postoperative recurrence of PSP.

\section{Acknowledgments}

None.

\section{Footnote}

Conflicts of Interest: The authors have no conflicts of interest to declare.

Etbical Statement: The authors are accountable for all aspects of the work in ensuring that questions related to the accuracy or integrity of any part of the work are appropriately investigated and resolved. This study was approved by the institutional review board of Takasago Municipal Hospital (No. 2018-3), and informed consent was obtained through the hospital website with an opt-out option.

\section{References}

1. Tsuboshima K, Wakahara T, Matoba Y, et al. Pleural coating by $50 \%$ glucose solution reduces postoperative recurrence for spontaneous pneumothorax. Ann Thorac Surg 2018;106:184-91.

2. Hirai K, Kawashima T, Takeuchi S, et al. Covering the staple line with a polyglycolic acid sheet after bullectomy for primary spontaneous pneumothorax prevents postoperative recurrent pneumothorax. J Thorac Dis 2015;7:1978-85.

3. Hong KP, Kim DK, Kang KH. Staple Line Coverage with a Polyglycolic Acid Patch and Fibrin Glue without Pleural Abrasion after Thoracoscopic Bullectomy for Primary Spontaneous Pneumothorax. Korean J Thorac Cardiovasc Surg 2016;49:85-91.

4. Lee $\mathrm{S}$, Kim HR, Cho S, et al. Staple line coverage after bullectomy for primary spontaneous pneumothorax: a randomized trial. Ann Thorac Surg 2014;98:2005-11.

5. Rena O, Massera F, Papalia E, et al. Surgical pleurodesis for Vanderschueren's stage III primary spontaneous pneumothorax. Eur Respir J 2008;31:837-41.

6. Cardillo G, Carleo F, Giunti R, et al. Videothoracoscopic talc poudrage in primary spontaneous pneumothorax: A single-institution experience in 861 cases. J Thorac Cardiovasc Surg 2006;131:322-8.

7. Sawabata N, Ohta M, Matsumura A, et al. Optimal distance of malignant negative margin in excision of nonsmall cell lung cancer: A multicenter prospective study. Ann Thorac Surg 2004;77:415-20.

8. Mohiuddin K, Haneuse S, Sofer T, et al. Relationship between margin distance and local recurrence among patients undergoing wedge resection for small $(\leq 2 \mathrm{~cm})$ non-small cell lung cancer. J Thorac Cardiovasc Surg 2014;147:1169-75.

9. Wolf AS, Swanson SJ, Yip R, et al. The Impact of Margins on Outcomes After Wedge Resection for Stage I Non-Small Cell Lung Cancer. Ann Thorac Surg 2017;104:1171-8.

10. Baumann MH, Strange C, Heffner JE, et al. Management of spontaneous pneumothorax: an American College of Chest Physicians Delphi consensus statement. Chest 2001;119:590-602.

11. MacDuff A, Arnold A, Harvey J. Management of spontaneous pneumothorax: British Thoracic Society Pleural Disease Guideline 2010. Thorax 2010;65 Suppl 2:ii18-31.

12. Tschopp JM, Bintcliffe O, Astoul P, et al. ERS task force statement: diagnosis and treatment of primary spontaneous pneumothorax. Eur Respir J 2015;46:321-35.

13. Tsuboshima K, Wakahara T, Matoba Y, et al. Singleincision thoracoscopic surgery using a chest wall pulley for lung excision in patients with primary spontaneous pneumothorax. Surg Today 2015;45:595-9.

14. Tsuboshima K, Nagata M, Wakahara T, et al. Feasibility of single-incision thoracoscopic surgery using a modified chest wall pulley for primary spontaneous pneumothorax: a propensity score matching analysis. Surg Today 2017;47:1129-34.

15. Katayama H, Kurokawa Y, Nakamura K, et al. Extended Clavien-Dindo classification of surgical complications: Japan Clinical Oncology Group postoperative complications criteria. Surg Today 2016;46:668-85.

16. Choi SY, Kim DY, Suh JH, et al. New bullae formation in the staple line increases the risk of recurrent pneumothorax following video-assisted thoracoscopic surgery bullectomy for primary spontaneous 
pneumothorax. J Thorac Dis 2018;10:4287-92.

17. Nishio W, Yoshimura M, Maniwa Y, et al. Re-Assessment of Intentional Extended Segmentectomy for Clinical

Cite this article as: Tsuboshima K, Matoba Y, Wakahara T. Optimal margin distance of bullectomy for primary spontaneous pneumothorax reduces postoperative recurrence. J Thorac Dis 2019;11(12):5115-5123. doi: 10.21037/jtd.2019.12.06
T1aN0 Non-Small Cell Lung Cancer. Ann Thorac Surg 2016;102:1702-10. 